AIRWAY BIOLOGY

\title{
Increased circulating endothelial progenitor cells in patients with bacterial pneumonia: evidence that bone marrow derived cells contribute to lung repair
}

\author{
M Yamada, H Kubo, K Ishizawa, S Kobayashi, M Shinkawa, H Sasaki
}

Thorax 2005;60:410-413. doi: 10.1136/thx.2004.034058

See end of article for authors' affiliations

Correspondence to:

Dr H Kubo, Department of

Geriatric and Respiratory

Medicine, Tohoku

University School of

Medicine, 1-1

Seiryoumachi, Aobaku,

Sendai 980-8574, Japan

hkubo@geriat.med.

tohoku.ac.jp

Received 30 August 2004

Accepted

15 November 2004

\begin{abstract}
Background: Tissue repair often occurs in organs damaged by various inflammatory diseases including pneumonia. Inflammatory stimuli induce a rapid and massive release of inflammatory cells from the bone marrow. Recent studies have suggested that bone marrow cells have the potential to differentiate into a variety of cell types. It has been shown that administration of lipopolysaccharide (LPS) to murine lungs induces a rapid release of endothelial progenitor cells (EPCs) into the circulation, and that bone marrow derived progenitor cells including EPCs contribute to lung repair after lung injury in mice. This study was undertaken to investigate the mobilisation of EPCs in humans following acute pneumonia.

Methods: Peripheral blood mononuclear cells (PBMCs) were isolated from venous blood taken from 23 patients with pneumonia during both the acute and convalescent phase. $1 \times 10^{6}$ PBMCs were plated on fibronectin coated culture slides and cultured in culture medium for endothelium. The numbers of EPCs were counted 8 days after plating.

Results: The number of circulating EPCs significantly increased in patients with pneumonia $(p<0.0001)$. Patients with low EPC counts tended to have persistent fibrotic changes in their lungs even after their recovery from pneumonia.

Conclusions: Inflammatory stimuli induce a rapid release of EPCs into the circulation in humans. A sufficient number of EPCs is necessary for proper lung repair following bacterial pneumonia.
\end{abstract}

1 nflammatory stimuli rapidly induce a massive release of inflammatory cells from the bone marrow. ${ }^{1}$ These inflammatory cells are important for host defence and resolution of an inflammatory response. It has recently become recognised that the bone marrow is a source of progenitor cells for several cell types ${ }^{2}$ including endothelial ${ }^{3}$ and epithelial cells. ${ }^{4}$ We have previously shown that administration of lipopolysaccharide (LPS) to murine lungs induced a rapid release of endothelial progenitor cells (EPCs) into the circulation and that bone marrow derived progenitor cells, including EPCs, contributed to lung repair after LPS induced lung injury in mice. ${ }^{5}$ In the present study we investigated the number of EPCs in the peripheral blood of patients with pneumonia to determine whether lung inflammation caused by disease processes such as bacterial pneumonia also induces a rapid release of EPCs into the circulation in humans.

\section{METHODS \\ Patients}

Twenty three adult patients with moderately severe community acquired bacterial pneumonia diagnosed by symptoms, physical examinations, laboratory data, sputum culture, and chest radiography in accordance with the Japanese Respiratory Society guidelines for the management of community acquired pneumonia were studied. ${ }^{6}$ Community acquired pneumonia was defined as an acute respiratory tract illness associated with radiographic shadowing on a chest radiograph which was neither pre-existing nor of any other known cause. All patients were assessed for severity using the pneumonia severity index (PSI) ${ }^{7}$ and CURB-65 score. ${ }^{8}$ High resolution computed tomography (HRCT) scans were performed to determine the range of pneumonia before the start of treatment and to identify persistent fibrotic changes 8 weeks after treatment was completed. Two radiologists evaluated the HRCT scan images. We defined the linear and reticular opacity based on HRCT scan images as fibrotic change. Subjects were excluded if their clinical course was suggestive of atypical pneumonia. We also excluded subjects with cardiovascular disease, cancer, or diabetes because these diseases have been reported to influence circulating EPC counts. ${ }^{7}$ All subjects signed an informed consent document approved by the local ethics committee of the Tohoku University School of Medicine.

\section{Cell culture assay for circulating EPCs}

Isolation of EPCs was performed mainly according to the method previously reported. ${ }^{39}{ }^{10}$ Briefly, a $10 \mathrm{ml}$ sample of venous blood was collected from the patients to isolate EPCs. Blood was collected before treatment and 8 weeks after treatment was completed. At the time of the first sampling the duration of the illness was less than 28 hours (median 24 hours; range 17-28 hours). Samples were processed within 6 hours of collection and peripheral blood mononuclear cells (PBMCs) were isolated by density gradient centrifugation using Ficoll (Amersham Biosciences, Piscataway, NJ, USA). Isolated cells were suspended in endothelial growth medium and $10^{6}$ cells/well were plated on 4-well culture slides coated with fibronectin (Biocoat, BD Labware, Franklin Lakes, NJ, USA). The number of EPCs per $\mathrm{mm}^{2}$ on the culture slide was counted 8 days after plating and, after determining the number of PBMCs in $10 \mathrm{ml}$ peripheral blood and the number of EPCs in $10^{6}$ mononuclear cells, we also calculated the number of EPCs obtained from $1 \mathrm{ml}$ of blood. Confirmation of endothelial cell lineage was

Abbreviations: CRP, C-reactive protein; EPC, endothelial progenitor cell; LPS, lipopolysaccharide; PBMC, peripheral blood mononuclear cell; PSI, pneumonia severity index 
performed by double positive staining for 1,1-dioctadecyl3,3,3', $3^{\prime}$-tetramethylin-docarbocyanin labelled acetylated low density lipoprotein (Dil-acLDL; $2.4 \mu \mathrm{g} / \mathrm{ml}$; Sigma, St Louis, MO, USA) and FITC-labelled lectin (10 $\mu \mathrm{g} / \mathrm{ml} ;$ Sigma). We also performed immunostaining against vascular endothelial growth factor receptors 2 and CD31 which are considered to be markers of endothelial cell lineage.

\section{Analysis of data}

Values are expressed as median (25-75th interquartile range, IQR) unless otherwise indicated. Individual comparisons were performed using the non-parametric Wilcoxon signed rank test. Correlations between the number of EPCs before treatment and white blood cell counts, C-reactive protein (CRP), age, PSI, CURB-65 score, blood monocyte counts, and blood lymphocyte counts were analysed with the Spearman rank correlation test. The non-parametric Wilcoxon rank sum test was used for comparisons of continuous variables and Fisher's exact test for comparisons of categorical variables between the groups with low and high EPC counts.

\section{RESULTS AND DISCUSSION}

\section{Characteristics of patients}

Twenty three adult patients with moderately severe community acquired bacterial pneumonia were enrolled in the study (table 1). The mean age of the patients was 73.0 years (median 76, range 30-95, IQR 65-80). None of the patients had coexisting illnesses such as neoplastic disease, cardiovascular disease, diabetes, liver disease, cerebrovascular disease, or renal disease. None were receiving concurrent medication or had received prior antibiotic treatment, and none were smokers. All patients were febrile at the time of the first sampling (median $38.3^{\circ} \mathrm{C}$, range $37.6-38.9$ ). The extent of the radiographic changes due to pneumonia was limited to a single lobe in all patients. The mean initial PSI was 78.6 (median (76 (IQR 65-92)). Four patients had a CURB-65 score of 0 , six had a score of 1 , and 13 had a score of 2. Bacterial examinations of the sputum revealed Streptococcus pneumoniae in six patients, Haemophilus influenzae in two and Moraxella catarrhalis in one patient. In 14 patients we could not identify a causal organism.

\section{Circulating EPCs at acute phase in patients with pneumonia}

Flat and spindle-like attaching cells appeared in cultured PBMCs. The morphological appearance of attaching cells resembled that of EPCs previously reported. ${ }^{3}{ }^{10}$ We further defined that double positive cells for Dil-acLDL incorporation and lectin staining, which were reported as endothelial cell lineage markers, were EPCs. Figure 1 shows representative images of EPCs in pre-treatment (fig lA-D) and posttreatment (fig $\mathrm{lE}-\mathrm{H}$ ) samples from a patient with pneumonia.

As shown in fig II, the median (25-75th IQR) number of EPCs $/ \mathrm{mm}^{2}$ on the culture slide was significantly higher in pre-treatment samples than in post-treatment samples (672 (433-862) v 283 (213-372)). The median (25-75th IQR) number of EPCs/ml blood was also higher in pre-treatment samples than in post-treatment samples (2298 (1602-3820) $\mathrm{v}$ 752 (513-1186); fig $1 \mathrm{~J}$ ). These data suggest that inflammatory stimuli induce the release of EPCs into the circulation in patients with bacterial pneumonia, just as previously reported in mice. ${ }^{5}$

We then determined whether the number of EPCs correlated with other factors including pre-treatment laboratory data. The number of EPCs was not significantly correlated with peripheral blood white blood cell (WBC) counts or CRP, which are considered indicators of the extent of inflammation (WBC counts: $\rho=0.387, p=0.1692$; CRP: $\rho=0.009, p=0.9649$ ). The severity of pneumonia (PSI, CURB-65) was also not significantly correlated with the number of EPCs (PSI: $\rho=-0.247, \mathrm{p}=0.2461$; CURB-65: $\rho=-0.190, p=0.3722$ ). We also observed a weak inverse correlation between the subjects' age and the number of EPCs, but this relationship was not statistically significant $(\rho=-0.255, p=0.1016)$. Interestingly, there was a strong correlation between peripheral blood monocyte counts and the number of EPCs $(\rho=0.635, p=0.0029$; fig $1 \mathrm{~K})$. We also observed a correlation between peripheral blood lymphocyte counts and the number of EPCs $(\rho=0.784, p=0.0002$; fig $1 \mathrm{~L}$ ). The finding that pre-treatment EPC counts in our patients were strongly correlated with monocyte and lymphocyte counts suggests that mechanisms of EPC mobilisation from bone marrow may resemble mechanisms of monocyte and lymphocyte mobilisation-mechanisms that involve mediators such as stromal cell derived factor 1/CXCR4 axis. ${ }^{11}$

Lung fibrotic changes in patients with low EPC counts We divided the subjects into two groups based on their pretreatment EPC counts to analyse the relationship between EPC counts and the clinical characteristics of the patients (table 1). The EPC count was not associated with sex, age, peripheral blood WBC counts, serum CRP, serum albumin,

Table 1 Characteristics of patients according to EPC counts $(n=23)^{*}$

\begin{tabular}{|c|c|c|c|c|}
\hline Characteristic & $\begin{array}{l}\text { All subjects } † \\
(\mathrm{~N}=23)\end{array}$ & $\begin{array}{l}\text { Low EPC groupt } \\
(\mathrm{N}=11)\end{array}$ & $\begin{array}{l}\text { High EPC groupt } \\
(\mathrm{N}=12)\end{array}$ & p value \\
\hline $\operatorname{Sex}(M: F)$ & $12: 11$ & $5: 6$ & $6: 6$ & 0.99 \\
\hline Age (years) & $76(65-80)$ & $77(68-82)$ & $73(63-79)$ & 0.31 \\
\hline WBC $\left(\times 10^{3} / \mathrm{mm}^{3}\right)$ & $11.7(8.2-14.0)$ & $11.7(7.9-12.2)$ & $13.0(10.5-15.0)$ & 0.13 \\
\hline $\mathrm{Hb}(\mathrm{g} / \mathrm{dl})$ & $11.4(10.9-12.1)$ & $11.2(10.9-11.9)$ & $11.6(10.8-12.3)$ & 0.51 \\
\hline Albumin $(\mathrm{g} / \mathrm{dl})$ & $3.4(3.1-4.0)$ & $3.2(2.7-3.7)$ & $3.6(3.2-4.0)$ & 0.10 \\
\hline CRP (mg/dl) (normal range 0-0.5) & $11.5(6.4-20.6)$ & $11.4(6.4-19.4)$ & $12.6(7.2-21.6)$ & 0.69 \\
\hline $\mathrm{SpO}_{2}(\%)$ & $93(89-93)$ & $93(90-93)$ & $93(90-94)$ & 0.60 \\
\hline PSI & $76(65-92)$ & 77 (69-97) & $71(63-92)$ & 0.41 \\
\hline \multicolumn{5}{|l|}{ CURB-65 score } \\
\hline 0 or 1 & 10 & 5 & 5 & 0.99 \\
\hline 2 & 13 & 6 & 7 & \\
\hline Patients with persistent fibrotic changes & 7 & 6 & 1 & 0.027 \\
\hline
\end{tabular}

$\mathrm{CRP}, \mathrm{C}$-reactive protein; $\mathrm{Hb}$, haemoglobin; PSI, pneumonia severity index.

* Values are expressed as median (25-75th IQR) unless otherwise indicated.

†Pretreatment EPC counts: 2298 (1602-3820)/ml blood in all subjects, $1523(1039-2206) / \mathrm{ml} \mathrm{blood} \mathrm{in} \mathrm{the} \mathrm{low} \mathrm{EPC} \mathrm{group,} \mathrm{and} \mathrm{3687} \mathrm{(2656-4788)/ml} \mathrm{blood} \mathrm{in}$ the high EPC group.

$\ddagger$ Non-parametric Wilcoxon rank sum test was used for comparisons of continuous variables and Fisher's exact test was used for comparisons of categorical variables. 


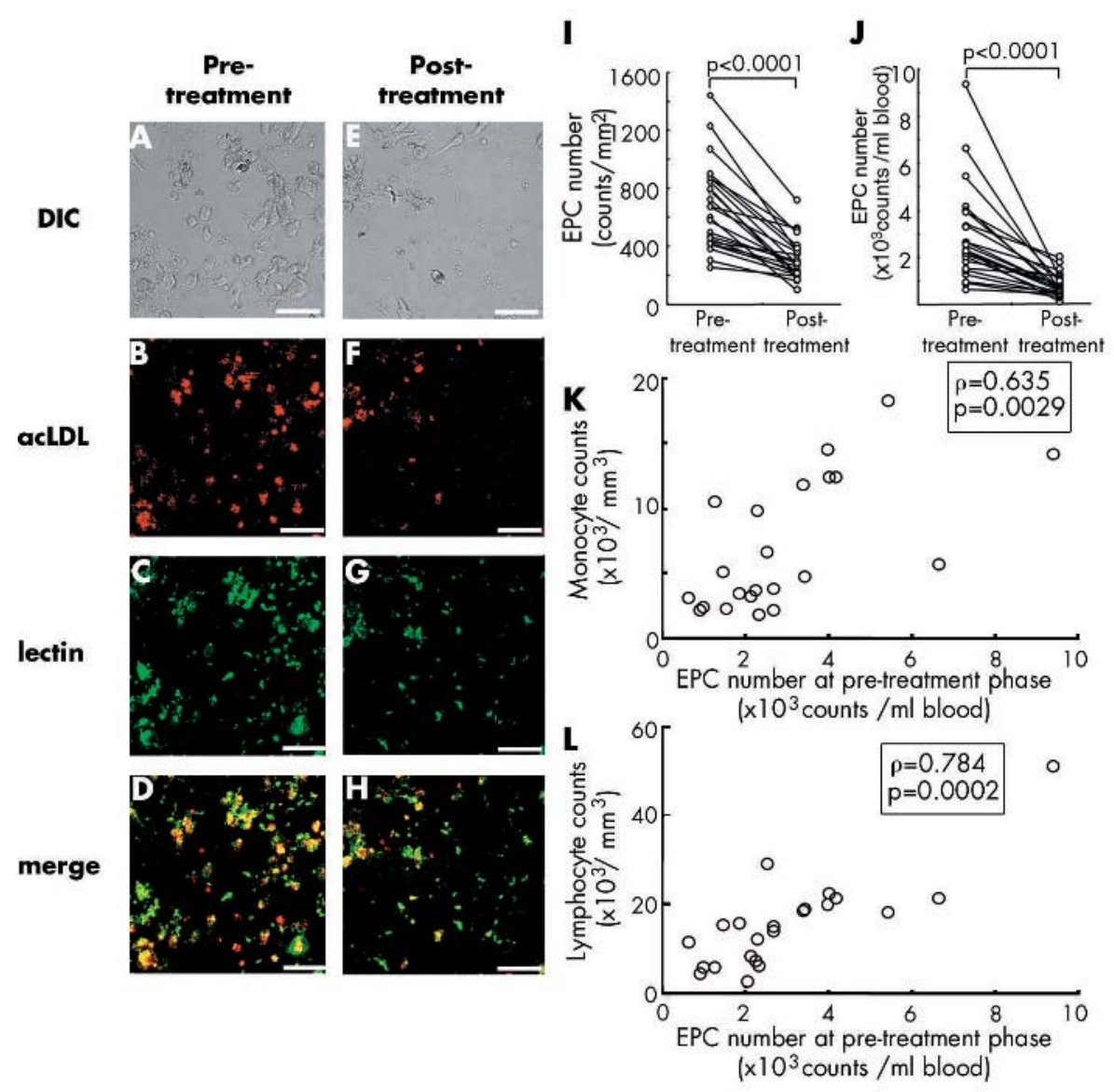

Figure 1 Circulating endothelial progenitor cells (EPCs) were increased at the acute phase in patients with pneumonia. Representative images of EPCs in pre-treatment $(A-D)$ and post-treatment $(E-H)$ samples from a patient with pneumonia. Differential interference contrast $(D I C)$ images $(A, E)$, acLDL uptake (B, F; red), lectin binding (C, G; green), and acLDL uptake/lectin binding overlay images (D, H; yellow) of EPCs are shown. The number of EPCs at the pre-treatment phase was significantly higher than at the post-treatment phase. Scale bars $25 \mu \mathrm{m}$. (I, J) Pre-treatment and post-treatment EPCs were isolated from the peripheral blood of 23 patients with bacterial pneumonia. (I) Number of EPCs per $\mathrm{mm}^{2}$ and (J) number of EPCs per $\mathrm{ml}$ blood after adjustment for the number of mononuclear cells in peripheral blood. p values were calculated with the non-parametric Wilcoxon signed rank test. Correlation between the number of EPCs and (K) peripheral blood monocytes or (L) lymphocytes in patients with pneumonia showing Spearman's rho ( $\rho)$ and $p$ values.

percutaneous oxygen saturation, or the severity of pneumonia (PSI, CURB-65). Similarly, EPC counts were not significantly associated with the infecting organism (data not shown).

We also analysed the relation between EPC counts and the patients' state after recovery from pneumonia. Interestingly, patients with low pre-treatment EPC counts tended to have persistent fibrotic changes in their lung, based on HRCT scan images, even after they had recovered. We have previously shown that suppression of bone marrow derived progenitor cells, including EPCs, caused incomplete repair from LPS induced lung injury. ${ }^{5}$ Several reports have suggested that EPC counts could provide a marker of vascular function in ischaemic stress. ${ }^{91213}$ Although the nature and size of the present study does not permit us to determine whether EPC levels can accurately predict the adequacy of repair of lung injury caused by pneumonia, the data suggest that bone marrow derived progenitor cells, including EPCs, contribute to lung repair following bacterial pneumonia, and that the levels of EPCs may predict the ability for lung repair following injury.

In this study we have shown that there is a strong correlation between peripheral blood lymphocyte counts and the number of EPCs. It is widely known that lymphocytopenia is a poor prognostic factor in infectious diseases including bacterial pneumonia. ${ }^{14}{ }^{15}$ Because our data showed that suppression of EPCs might cause incomplete repair from pneumonia, we speculate that a poor prognosis in pneumonia patients with lymphocytopenia may be due to a reduction in the ability of the lung to repair that is thought to be correlated with EPC counts.

\section{Authors' affiliations}

M Yamada, H Kubo, K Ishizawa, S Kobayashi, M Shinkawa, H Sasaki, Department of Geriatric and Respiratory Medicine, Tohoku University School of Medicine, Sendai, 980-8574, Japan

This work was supported by a grant from the Japan Society for the Promotion of Science No 13670589 to HK.

The sponsor of the study had no role in study design, data collection, data analysis, data interpretation, or the writing of the report.

\section{REFERENCES}

1 Kubo H, Graham L, Doyle NA, et al. Complement fragment-induced release of neutrophils from bone marrow and sequestration within pulmonary capillaries in rabbits. Blood 1998;92:283-90. 
2 Krause DS. Plasticity of marrow-derived stem cells. Gene Ther 2002:9:754-8.

3 Asahara T, Murohara T, Sullivan A, et al. Isolation of putative progenitor endothelial cells for angiogenesis. Science 1997;275:964-7.

4 Kotton DN, Ma BY, Cardoso WV, et al. Bone marrow-derived cells as progenitors of lung alveolar epithelium. Development 2001; 128:5181-8.

5 Yamada M, Kubo H, Kobayashi S, et al. Bone marrow-derived progenitor cells are important for lung repair after lipopolysaccharide-induced lung injury. J Immunol 2004;172:1266-72.

6 Yanagihara K, Kohno S, Matsusima T. Japanese guidelines for the management of community-acquired pneumonia. Int $J$ Antimicrob Agents 2001; 18:S45-8

7 Fine MJ, Auble TE, Yealy DM, et al. A prediction rule to identify low-risk patients with community-acquired pneumonia. N Engl J Med 1997:336:243-50.

8 Lim WS, van der Eerden MM, Laing $R$, et al. Defining community acquired pneumonia severity on presentation to hospital: an international derivation and validation study. Thorax 2003;58:377-82
9 Hill JM, Zalos G, Halcox JP, et al. Circulating endothelial progenitor cells, vascular function, and cardiovascular risk. N Engl J Med 2003;348:593-600

10 Shintani S, Murohara T, Ikeda H, et al. Mobilization of endothelial progenitor cells in patients with acute myocardial infarction. Circulation $2001 ; 103: 2776-9$

11 Yamaguchi J, Kusano KF, Masuo O, et al. Stromal cell-derived factor-1 effects on ex vivo expanded endothelial progenitor cell recruitment for ischemic neovascularization. Circulation 2003;107:1322-8.

12 Vasa M, Fichtlscherer S, Aicher A, et al. Number and migratory activity of circulating endothelial progenitor cells inversely correlate with risk factors for coronary artery disease. Circ Res 2001;89:e1-7.

13 Taguchi A, Matsuyama T, Moriwaki H, et al. Circulating CD34-positive cells provide an index of cerebrovascular function. Circulation 2004; 109:2972-5.

14 Lewis RT, Klein H. Risk factors in postoperative sepsis: significance of preoperative lymphocytopenia. J Surg Res 1979:26:365-71.

15 Proust J, Rosenzweig P, Debouzy C, et al. Lymphopenia induced by acute bacterial infections in the elderly: a sign of age-related immune dysfunction of major prognostic significance. Gerontology 1985;31:178-85.

\section{LUNG ALERT}

Asthma deaths and bronchodilator therapy

A Anderson HR, Ayres JG, Sturdy PM, et al. Bronchodilator treatment and deaths from asthma: case-control study. BMJ 2005;330:117-20

his study concerning asthma deaths and bronchodilator therapy covered 33 health boards in the UK (27\% of population) for 4 years from 1994 to 1998 . Patients had asthma as the principal cause of death (at certification), were under the age of 65, with no other underlying non-respiratory cause. Data were gathered for the 5 year period before death using hospital and primary care sources. This group was then matched with controls from the local hospital discharged with a diagnosis of asthma within the same time period and of the same age. When matching for severity, the age of onset of asthma, presence of chronic obstructive disease, previous hospital admissions for asthma, and the number of medications prescribed were used. The total number of deaths included was $532(22 \%$ were excluded), of which $44 \%$ occurred in hospital. It was noted that those who died had an earlier age of onset of asthma, mention of obesity, and evidence of associated chronic obstructive pulmonary disease.

Short acting $\beta_{2}$ agonist treatment prescribed 1-5 years before death was associated with an increased risk of mortality (odds ratio $2.05,95 \%$ CI 1.26 to $3.33, p<0.01$ ). No such association was noted during the year preceding death. Inhaled corticosteroids showed no evidence of an altered risk of mortality, but there was a suggestion that long acting $\beta_{2}$ drugs may be associated with a decrease in mortality risk. A number of possible explanations may underlie the observed association between short acting $\beta_{2}$ agonist therapy and mortality, only one of which is a direct toxic effect of the drugs themselves.

Locum Registrar, Royal London Hospital, London, UK; graniaprice@doctors.org.uk 